Yoshio Kato

Nagoya Math. J.

Vol. 54 (1974), 7-20

\title{
ON A CLASS OF NON-ELLIPTIC BOUNDARY PROBLEMS
}

\section{Dedicated to Professor Minoru Kurita on his 60th birthday}

\author{
YOSHIO KATO
}

\section{Introduction.}

Let $\Omega$ be a bounded domain in $R^{l}(l \geq 2)$ with $C^{\infty}$ boundary $\Gamma$ of dimension $l-1$ and let there be given a second order elliptic differential equation

$$
A u=-\sum_{i, j=1}^{l} \partial_{i}\left(a_{i j} \partial_{j} u\right)+\sum_{i=1}^{l} a_{i} \partial_{i} u+\alpha u=f \quad \text { in } \Omega,
$$

where $\partial_{j}=\partial / \partial x_{i}$ and all coefficients are assumed, for the sake of simplicity, to be real-valued and $C^{\infty}$ on $\bar{\Omega}=\Omega^{\cup} \Gamma$. It is also assumed that $a_{i j}=a_{j i}$ on $\Omega$ and that there exists a positive constant $c_{0}$ such that

$$
\sum_{i, j=1}^{l} a_{i j}(x) \xi_{i} \xi_{j} \geq c_{0}|\xi|^{2}
$$

holds for all $x \in \bar{\Omega}$ and $\xi \in \boldsymbol{R}^{l}$.

Then we consider a boundary condition

$$
B u=\alpha \partial_{\nu} u+\gamma u+\beta u=\varphi \text { on } \Gamma,
$$

where $\alpha, \beta$ are real-valued $C^{\infty}$ functions on $\Gamma, \gamma$ is a $C^{\infty}$ real vector field tangent to $\Gamma$, and $\partial_{\nu} u$ denotes the conormal derivative of $u$, i.e.,

$$
\partial_{\nu} u=\sum_{i, j=1}^{l} a_{i j} n_{i} \partial_{j} u,
$$

$n=\left(n_{1}, \cdots, n_{l}\right)$ being the exterior normal of $\Gamma$. Moreover, throughout this paper, we assume $\alpha \geq 0$ on $\Gamma$.

In case $\gamma=0$ on $\Gamma$, the boundary problem (1)-(2) was discussed in $[2,3]$ by using the Hilbert space technique and the elliptic regularization. This paper is a continuation of their studies and is especially nothing but a slight improvement of [2].

Received December 13, 1973. 
Now we state the results obtained. The notations appearing will be made clear in $\S 1$.

THEOREM 1. If we assume that

$$
\frac{1}{2} \gamma^{*}(1)+\beta>0 \quad \text { on } \quad \Gamma_{0}=\{x \in \Gamma ; \alpha(x)=0\},
$$

it then follows that for every $f \in H^{k-2}(\Omega ; p)$ and every $\varphi \in H^{k-1}(\Gamma)(k$ integer $\geq 2$ ), the boundary problem

$$
\begin{cases}(A+\lambda) u=f & \text { in } \Omega \\ (B+t) u=\varphi & \text { on } \Gamma\end{cases}
$$

has the unique solution $u$ in $H^{k}(\Omega ; p)$, provided $\lambda \geq \lambda_{0}$, a number which is a constant not depending on $k$, and $t \geq t_{k}$, a number which is a constant depending in general on $k$.

Moreover it follows that there exists a constant $C_{k}>0$ independent of $t \geq t_{k}$ such that

$$
\|u ; p\|_{k} \leq C_{k}\left(\|f ; p\|_{k-2}+\|\varphi\|_{k-1, \Gamma}\right) .
$$

CoRollary. Assume, in addition to (3), that

$$
\gamma=0 \quad \text { in a neighbourhood of } \Gamma_{0} \text {. }
$$

Then we can take as $t_{k}=0$ for every $k$.

The following example shows us that condition (6) is necessary for Theorem 1 to be valid for $t_{k}=0$.

ExAmple. Let $\Omega$ be a bounded domain in the $(x, y)$-plane whose boundary $\Gamma$ is a $C^{\infty}$ curve and contains an open interval $\omega \ni(0,0)$ in the $x$-axis. In (1) and (2) we take as $A=\Delta, \alpha=0$ in $\omega, \gamma=-x \partial / \partial x$ in $\omega, \beta \geq 1$ integer and $\varphi=\alpha \partial v / \partial n+\gamma v+\beta v$, where $v$ is a harmonic function whose boundary value is $C^{\infty}$ except the origin and is equal to $|x|^{\beta}$ in $\omega$. Clearly we have $\varphi \in C^{\infty}(\Gamma)$.

Then $u=v$ is a solution belonging to $C^{\beta-1}(\bar{\Omega})$ of the problem

$$
\begin{cases}-\Delta u=0 & \text { in } \Omega \\ \alpha \frac{\partial u}{\partial n}+\gamma u+\beta u=\varphi & \text { on } \Gamma,\end{cases}
$$

but does not belong to $C^{\beta}(\bar{\Omega})$. Here it is easily seen that (3) is satisfied 
but not (6).

THEOREM 2. If $\Gamma_{0}$ is a $C^{\infty}$ manifold of dimension $l-2$ and $\gamma$ is transversal to $\Gamma_{0}$, it then follows that for every $f \in H^{k-2}(\Omega ; p)$ and every $\varphi \in H^{k-1}(\Gamma)$ ( $k$ integer $\geq 2$ ) the problem (4) with $t=0$ has the unique solution $u$ in $H^{k}(\Omega ; p)$, provided $\lambda \geq \lambda_{1}$ which is a constant not depending on $k$. Moreover the $u$ satisfies (5).

In case $\beta=0$, this is nothing but a class of the oblique derivative problems, which was already discussed in [1] by the slightly different manner (cf. $\S 7$ of [1]).

The plan of the paper is as follows. $\S 1$ is devoted to preliminaries of the proof of Theorem 1, which will be given in $\S 2$. Corollary and Theorem 2 will be briefly proved in $\S \S 3$ and 4 , respectively, by the similar argument as in Theorem 1.

\section{§1. Preliminaries.}

Let $\gamma$ be a $C^{\infty}$ real vector field tangent to $\Gamma$. The adjoint $\gamma^{*}$ of $\gamma$ is defined by the identity

$$
\int_{\Gamma} \gamma u \cdot v d \sigma=\int_{\Gamma} u \cdot \gamma^{*} v d \sigma, \quad u, v \in C^{\infty}(\Gamma)
$$

where $d \sigma$ is the Lebesque measure on $\Gamma$.

Let $\left\{U_{j}\right\}, j=1, \cdots, N$, be a family of open subsets of $\boldsymbol{R}^{l}$, covering $\Gamma$, and assume that there exists a $C^{\infty}$ coordinate transformation $y=\kappa_{j}(x)$ on $U_{j}$ such that $\Omega \cap U_{j}$ is mapped in a one-to-one way onto an open portion $\Sigma_{j}$ of a half space $y_{l}<0$ and $\Gamma_{j}=\Gamma \cap U_{j}$ is transformed onto an open portion $\tau_{j}$ of $y_{l}=0$. Moreover assume that $d y=J_{j} d x$ and $\left.d \sigma=K_{j} d y^{\prime}\left(y^{\prime}=y_{1}, \cdots, y_{l-1}\right)\right)$.

Let $\left\{\zeta_{j}(x)\right\}$ be a partition of unity of $\Gamma$ belonging to $\left\{U_{j}\right\}$, i.e., $\zeta_{j} \in C_{0}^{\infty}\left(U_{j}\right), \zeta_{j} \geq 0$ and $\sum_{j=1}^{N} \zeta_{j}(x)=1$ on $\Gamma$. Using the partition of unity $\left\{U_{j}, \zeta_{j}\right\}$, we can easily prove

Lemma 1. There exists a $C^{\infty}$ function $b(x)$ on $\Gamma$ such that $\gamma^{*}=$ $-\gamma+b(x)$.

Proof. We assume that by the transformation $\kappa_{j}$ the vector field $\gamma$ is altered to 


$$
\delta_{j}=\sum_{k=1}^{l-1} c_{j k} \partial_{k} \quad\left(\partial_{k}=\partial / \partial y_{k}\right)
$$

Then we have

$$
\begin{aligned}
\int_{\Gamma} \gamma u \cdot v d \sigma= & \sum_{j=1}^{N} \int_{\Gamma_{j}} \gamma\left(\zeta_{j} u\right) \cdot v d \sigma \\
= & \sum_{j} \int_{y_{l}=0} \sum_{k} c_{j k} \partial_{k}\left(\zeta_{j} u\right) \cdot v K_{j} d y^{\prime}=-\sum_{j} \int_{y_{l}=0} \sum_{k} \zeta_{j} u \cdot \partial_{k}\left(c_{j k} K_{j} v\right) d y^{\prime} \\
= & -\sum_{j} \int_{y_{l}=0} \sum_{k} \zeta_{j} u\left\{c_{j k} K_{j} \partial_{k} v+\partial_{k}\left(c_{j k} K_{j}\right) v\right\} d y^{\prime} \\
= & -\sum_{j} \int_{y_{l}=0} \zeta_{j} u \cdot \sum_{k}\left(c_{j k} \partial_{k} v\right) K_{j} d y^{\prime} \\
& -\sum_{j} \int_{y_{l}=0} \zeta_{j} u \cdot \sum_{k} \partial_{k}\left(c_{j k} K_{j}\right) K_{j}^{-1} v K_{j} d y^{\prime} \\
= & -\sum_{j} \int_{\Gamma} \zeta_{j} u \cdot \gamma v d \sigma-\sum_{j} \int_{\Gamma} u\left\{\zeta_{j} K_{j}^{-1} \sum \partial_{k}\left(c_{j k} K_{j}\right)\right\} v d \sigma
\end{aligned}
$$

which completes the proof.

The following lemma can be easily proved. So we omit the proof.

LEMMA 2. Under condition (3) we can find a function $q(x) \in C^{\infty}(\bar{\Omega})$ satisfying

(i) $q>0$ in $\Omega$ and $q=\alpha$ on $\Gamma$.

(ii) There exist two positive constants $C$ and $d$ such that $C \operatorname{dis}(x, \Gamma)$ $\leq q(x)$ in $\Omega_{d}=\{x \in \bar{\Omega} ; \operatorname{dis}(x, \Gamma)<d\}$.

(iii) There exists a positive constant $c_{1}$ such that

$$
\frac{1}{2} \partial_{\nu} q+\frac{1}{2} \gamma^{*}(1)+\beta \geq c_{1} \text { on } \Gamma \text {. }
$$

LEMMA 3. For any $\delta>0$ there exists a constant $C_{\delta}>0$ such that $\|u\|_{0, \Omega}^{2} \leq \delta\|p \partial u\|_{0, \Omega}^{2}+C_{\delta}\|p u\|_{0, \Omega}^{2}, \quad u \in C^{\infty}(\bar{\Omega})$,

where $p=\sqrt{q},\|u\|_{0, \Omega}^{2}=\int_{\Omega}|u|^{2} d x$ and

$$
\|p \partial u\|_{0, \Omega}^{2}=\sum_{j=1}^{l} \int q\left|\partial_{j} u\right|^{2} d x
$$

Proof. This lemma is due to [2]. Let $\zeta_{0}(x) \in C_{0}^{\infty}(\Omega)$ such that $\zeta_{0}=$ $1-\sum_{j=1}^{N} \zeta_{j}$ in $\Omega$ and $=0$ outside of $\bar{\Omega}$. Then $u=\sum_{j=1}^{N} \zeta_{j} u+\zeta_{0} u$ in $\Omega$. Hence we have 


$$
\begin{aligned}
\|u\|_{0, \Omega}^{2} & \leq\left(\sum_{j=1}^{N}\left\|\zeta_{j} u\right\|_{0, \Omega}+\left\|\zeta_{0} u\right\|_{0, \Omega}\right)^{2} \\
& \leq \text { const. }\left(\sum_{j=1}^{N} \int_{\Sigma_{j}}\left|v_{j}\right|^{2} d y+\|p u\|_{0, \Omega}^{2}\right),
\end{aligned}
$$

where $v_{j}=\sqrt{J_{j}} \zeta_{j} u$ is in $C_{0}^{\infty}\left(\Sigma_{j} \cup \tau_{j}\right)$. It was indicated by Hayashida in [2] that for any $\varepsilon>0$ the inequality

$$
\int_{\Sigma_{j}}\left|v_{j}\right|^{2} d y \leq \varepsilon \int_{\Sigma_{j}}\left|y_{l}\right|\left|\partial_{l} v_{j}\right|^{2} d y+\frac{1}{\varepsilon} \int_{\Sigma_{j}}\left|y_{l}\right|\left|v_{j}\right|^{2} d y
$$

holds. Thus we can establish the proof with the aid of Lemma 2 .

Now we introduce an integro-differential bilinear form:

$$
Q[u, v]=B[u, q v]+\int_{\Gamma}(\gamma u+\beta u) \cdot v d \sigma,
$$

where

$$
B[u, v]=\int_{\Omega}\left(\sum_{i, j=1}^{l} a_{i j} \partial_{i} u \cdot \partial_{j} u+\sum_{i=1}^{l} a_{i} \partial_{i} u \cdot v+a u \cdot v\right) d x .
$$

It is easily seen that $u \in C^{2}(\bar{\Omega})$ satisfies (1) and (2) if and only if it satisfies

$$
Q[u, v]=(q f, v)_{\Omega}+(\varphi, v)_{\Gamma}, \quad v \in C^{\infty}(\bar{\Omega}),
$$

where $(,)_{\Omega}$ and $(,)_{\Gamma}$ denote the usual inner products in $L^{2}(\Omega)$ and $L^{2}(\Gamma)$, respectively. Hence we have only to deal with (7). This idea was used in [4].

Throughout the paper we always assume condition (3).

Proposition 1. There exist two positive constants $c_{2}, \lambda_{0}$ such that

$$
Q_{\lambda}[u, u] \geq c_{2}\left(\|p \partial u\|_{0, \Omega}^{2}+\|p u\|_{0, \Omega}^{2}+\|u\|_{0, \Gamma}^{2}\right)
$$

holds for every $u \in C^{\infty}(\bar{\Omega})$ and $\lambda \geq \lambda_{0}$, where $\|u\|_{0, \Gamma}^{2}=(u, u)_{\Gamma}$ and

$$
Q_{\lambda}[u \cdot v]=Q[u, v]+\lambda(u, q v) .
$$

Proof. For $u \in C^{\infty}(\bar{\Omega})$ we have

$$
\begin{aligned}
Q[u, u]= & \int_{\Omega} q\left(\sum_{i, j=1}^{l} a_{i j} \partial_{i} u \cdot \partial_{j} \cdot u+\sum_{i=1}^{l} a_{i} \partial_{i} u \cdot u+a u u\right) d x \\
& +\frac{1}{2} \int_{\Omega} \sum_{i, j=1}^{l} a_{i j} \partial_{j} q \cdot \partial_{i}\left(u^{2}\right) d x+\int_{\Gamma}\left(\frac{1}{2} \gamma\left(u^{2}\right)+\beta u^{2}\right) d \sigma
\end{aligned}
$$




$$
\begin{aligned}
\geq & \frac{c_{0}}{2}\|p \partial u\|_{0, \Omega}^{2}-C\|p u\|_{0, \Omega}^{2}+\frac{1}{2} \int A_{0} q \cdot u^{2} d x \\
& +\int_{\Gamma}\left(\frac{1}{2} \partial_{\nu} q+\frac{1}{2} \gamma^{*}(1)+\beta\right) u^{2} d \sigma
\end{aligned}
$$

where $C$ is a constant and $A_{0}=-\sum_{i, j=1}^{l} \partial_{i} \alpha_{i j} \partial_{j}$. Thus, using Lemmas 2 and 3 , we can conclude the proposition.

For any $\varepsilon, 0<\varepsilon \leq 1$, putting $q_{\varepsilon}(x)=q(x)+\varepsilon$, we define an integrodifferential bilinear form as

$$
Q^{\varepsilon}[u, v]=B\left[u, q_{\varepsilon} v\right]+\int_{\Gamma}(\gamma u+\beta u) v d \sigma .
$$

Proposition 2. Let $\lambda \geq \lambda_{0}$ and $t \geq 0$. Then for every $f \in C^{\infty}(\bar{\Omega})$ and every $\varphi \in C^{\infty}(\Gamma)$, there exists the unique $u_{\varepsilon} \in C^{\infty}(\bar{\Omega})$ which depends also on $\lambda$ and $t$, satisfying

$$
Q_{\lambda, t}^{\varepsilon}\left[u_{\mathrm{e}}, v\right]=\left(q_{\mathrm{s}} f, v\right)_{\Omega}+(\varphi, v)_{\Gamma}, \quad v \in C^{\infty}(\bar{\Omega}) .
$$

Moreover it follows that there exists a constant $c_{3}>0$ independent of $\varepsilon, \lambda$ and $t$ such that

$$
c_{3}\left(\left\|p_{\varepsilon} \partial u_{\varepsilon}\right\|_{0, \Omega}^{2}+\left\|p_{\varepsilon} u_{\varepsilon}\right\|_{0, \Omega}^{2}+(1+t)\left\|u_{\varepsilon}\right\|_{0, \Gamma}^{2}\right) \leq\left\|p_{\varepsilon} f\right\|_{0, \Omega}^{2}+\|\varphi\|_{0, \Gamma}^{2},
$$

where $p_{\varepsilon}=\sqrt{q_{\varepsilon}}$ and

$$
Q_{\lambda, t}^{\varepsilon}[u, v]=Q^{\varepsilon}[u, v]+\lambda\left(u, q_{\varepsilon} v\right)+t(u, v)_{\Gamma} .
$$

Proof. By the same argument as in Proposition 1, we can immediately obtain

$$
\begin{gathered}
Q_{\lambda, t}^{\varepsilon}[u, u] \geq c_{2}^{\prime}\left(\left\|p_{\varepsilon} \partial u\right\|_{0, \Omega}^{2}+\left\|p_{\varepsilon} u\right\|_{0, \Omega}^{2}+(1+t)\|u\|_{0, \Gamma}^{2}\right) \\
\left(=c_{2}^{\prime}\|u\| \|_{\varepsilon, t}^{2}\right), \quad u \in C^{\infty}(\bar{\Omega}),
\end{gathered}
$$

with $c_{2}^{\prime}=\min \left(c_{2}, 1\right)$. Clearly we have

$$
\left\{\begin{array}{l}
Q_{\lambda, t}^{\varepsilon}[u, u] \geq \varepsilon c_{2}^{\prime}\|u\|_{1, \Omega}^{2} \\
\left|Q_{\lambda, t}^{\varepsilon}[u, v]\right| \leq \text { const. }\|u\|_{1, \Omega}\|v\|_{1, \Omega},
\end{array}\right.
$$

where

$$
\|u\|_{1, \Omega}^{2}=\sum_{j=1}^{l} \int_{\Omega}\left|\partial_{j} u\right|^{2} d x+\|u\|_{0, \Omega}^{2} .
$$

Accordingly we can apply the theorem of Riesz-Milgram-Lax which guarantees the existence of the unique solution $u_{\varepsilon}$ of (8) in $H^{1}(\Omega)$. It is 
well known that $u_{e}$ is really in $C^{\infty}(\bar{\Omega})$, since the problem is elliptic. In fact $u_{\varepsilon}$ satisfies

$$
\begin{cases}(A+\lambda) u_{\varepsilon}=f & \text { in } \Omega \\ (\alpha+\varepsilon) \partial_{\nu} u_{\varepsilon}+\gamma u_{\varepsilon}+(\beta+t) u_{\varepsilon}=\varphi & \text { on } \Gamma .\end{cases}
$$

Substituting $v=u_{s}$ in (8) and using (10), we obtain

$$
\begin{aligned}
c_{2}^{\prime}\left\|u_{\varepsilon}\right\|_{\varepsilon, t}^{2} & \leq Q_{\lambda, t}^{\varepsilon}\left[u_{\varepsilon}, u_{\varepsilon}\right]=\left(q_{\varepsilon} f, u_{\varepsilon}\right)_{\Omega}+\left(\varphi, u_{\varepsilon}\right)_{\Gamma} \\
& \leq\left\|p_{\varepsilon} f\right\|_{0, \Omega}\left\|p_{\varepsilon} u_{\varepsilon}\right\|_{0, \Omega}+\|\varphi\|_{0, \Gamma}\left\|u_{\varepsilon}\right\|_{0, \Gamma} \\
& \leq\left(\left\|p_{\varepsilon} f\right\|_{0, \Omega}+\|\varphi\|_{0, \Gamma}\right)\left\|u_{\varepsilon}\right\|_{\varepsilon, t},
\end{aligned}
$$

which proves (9).

Finally we shall define the Hilbert space $H^{k}(\Omega ; p)$ for integer $k \geq 0$. By $H^{s}(\Omega), s$ real, we denote the Sobolev space with norm $\|\cdot\|_{s, \Omega}$. Then $H^{k}(\Omega ; p)$ is a Hilbert space given by the completion of $C^{\infty}(\bar{\Omega})$ with respect to the norm $\|\cdot, p\|_{k}$ defined by

$$
\|u ; p\|_{k}^{2}=\left\|p \partial^{k} u\right\|_{0, \Omega}^{2}+\|u\|_{k-1 / 2, \Omega}^{2} .
$$

\section{Proof of Theorem 1 .}

Setting $U_{0}=\Omega-\bigcup_{k=1}^{N} U_{j}$, we obtain the partition of unity $\left\{U_{j}, \zeta_{j}\right\}$, $j=0,1, \cdots, N$, of $\bar{\Omega}$. In the following we denote by $U, \zeta, \kappa, \Sigma, \tau, J$ and $K$ one of $U_{j}, \zeta_{j}, \kappa_{j}, \Sigma_{j}, \tau_{j}, J_{j}$ and $K_{j}(j=1, \cdots, N)$, respectively, and assume that by the transformation $\kappa$ the form $Q_{\lambda, t}^{\varepsilon}[u \cdot v]$ is altered to, $\lambda$ fixed,

$$
\begin{aligned}
P_{t}^{\epsilon}[u, v]= & \int_{\Sigma}\left(\sum_{i, j=1}^{l} b_{i j} \partial_{i} u \cdot \partial_{j}\left(q_{\varepsilon} v\right)+\sum_{i=1}^{l} b_{i} \partial_{i} u \cdot q_{\varepsilon} v+b u q_{\varepsilon} v\right) d y \\
& +\int_{\tau} \delta u \cdot v K d y^{\prime}+\int_{\tau} \beta u v K d y^{\prime}+t \int_{\tau} u v K d y^{\prime} \\
= & \mathrm{I}[u, v]+\operatorname{II}[u, v]+\operatorname{III}[u, v]+\operatorname{IV}[u, v],
\end{aligned}
$$

with $b_{i j}=b_{j i}$. It then follows from (10) that there exists a constant $c_{2}^{\prime \prime}>0$ independent of $\varepsilon, \lambda$ and $t$ such that

$$
c_{2}^{\prime \prime}\left(\left\|p_{\varepsilon} \partial u\right\|_{0, \Sigma}^{2}+\left\|p_{\varepsilon} u\right\|_{0, \Sigma}^{2}+(1+t)\|u\|_{0, \Gamma}^{2}\right) \leq P_{t}^{\varepsilon}[u, u], \quad u \in C_{0}^{\infty}(U) .
$$

For any multi-integers $\rho=\left(\rho_{1}, \cdots, \rho_{l-1}\right)$ such that $|\rho|=\rho_{1}+\cdots+\rho_{l-1}$ $=r \geq 1$, we set

$$
T u=\partial^{\rho}(\zeta u)=\partial_{1}^{\rho_{1}} \cdots \partial_{l-1}^{\rho_{l-1}}(\zeta u)
$$

with $\partial_{j}=\partial / \partial y_{j}$. In the following propositions all constants are inde- 
pendent of $\varepsilon$ and $t \geq 0$.

Proposition 3. There exist positive constants $C_{\mathrm{I}}, C_{\mathrm{II}}$ and $C_{\mathrm{III}}$ depending only on the forms I, II and III, respectively, such that

$$
\begin{gathered}
P_{t}^{\mathrm{\epsilon}}[T u, T u]-P_{t}^{\mathrm{e}}\left[u, K^{-1} T^{*} K T u\right] \leq C_{\mathrm{I}}\left(\|u\|_{r, \Sigma}\left\|\partial\left(q_{\epsilon} T u\right)\right\|_{0, \Sigma}+\|u\|_{r, \Sigma}^{2}\right) \\
+C_{\mathrm{II}}\|u\|_{r, \tau}^{2}+C_{\mathrm{III}}\|u\|_{r-1, \tau}\|T u\|_{0, \tau}, \quad u \in C^{\infty}\left(\boldsymbol{R}_{y}^{n}\right),
\end{gathered}
$$

where $K\left(y^{\prime}, y_{l}\right)=K\left(y^{\prime}\right)$.

Proof. ( I ) Setting $R=b_{i j} \partial_{i}$ and $S=\partial_{j}$, and writting simply $(,)_{\Sigma}=($,$) and [A, B]=A B-B A$, we can compute as follows:

$$
\begin{aligned}
\left(R T u, S q_{\varepsilon} T u\right)= & \left(R u, T^{*} S q_{\varepsilon} T u\right)+\left([R, T] u, S q_{\varepsilon} T u\right) \\
= & \left(R u, T^{*} S q_{\varepsilon} K^{-1} K T u\right)+\left([R, T] u, S q_{\varepsilon} T u\right) \\
= & \left(R u, S q_{\varepsilon} K^{-1} T^{*} K T u\right)+\left(R u,\left[T^{*}, S q_{\varepsilon} K^{-1}\right] K T u\right) \\
& +\left([R, T] u, S q_{\varepsilon} T u\right) \\
= & \left(R u, S q_{\varepsilon} K^{-1} T^{*} K T u\right)+\left(R u,\left[T^{*}, S\right] q_{\varepsilon} T u\right) \\
& +\left([R, T] u, S q_{\varepsilon} T u\right)+\left(R u, S\left[T^{*}, q_{\varepsilon} K^{-1}\right] K T u\right)
\end{aligned}
$$

Thus

$$
\begin{aligned}
\mathrm{I}[T u, T u]-\mathrm{I}\left[u, K^{-1} T^{*} K T u\right] \leq & C\left(\|u\|_{r, \Sigma}\left\|\partial\left(q_{\varepsilon} T u\right)\right\|_{0, \Sigma}+\|u\|_{r, \Sigma}^{2}\right) \\
& +\int \sum_{\Sigma i, j=1}^{l} b_{i j} \partial_{i} u \cdot \partial_{j} v d y,
\end{aligned}
$$

where we put $v=\left[T^{*}, q_{\varepsilon} K^{-1}\right] K T u$. Now

$$
\begin{aligned}
(R u, S v)+(R v, S u)= & \left(R u,\left[T^{*}, q_{\varepsilon} K^{-1}\right] K T S u\right)+\left(R u,\left[S,\left[T^{*}, q_{\varepsilon} K^{-1}\right] K T\right] u\right) \\
& +\left(\left[T^{*}, q_{\varepsilon} K^{-1}\right] K T R u, S u\right)+\left(\left[R,\left[T^{*}, q_{\varepsilon} K^{-1}\right] K T\right] u, S u\right) \\
= & \left(R u,\left\{\left[T^{*}, q_{\varepsilon} K^{-1}\right] K T+T^{*} K\left[q_{\varepsilon} K^{-1}, T\right]\right\} S u\right)+O\left(\|u\|_{r, \Sigma}^{2}\right),
\end{aligned}
$$

which implies

$$
|(R u, S v)+(R v, S u)| \leq C\|u\|_{r, \Sigma}^{2} .
$$

This together with (14) and the fact $b_{i j}=b_{j i}$ implies

$$
\mathrm{I}[T u, T u]-\mathrm{I}\left[u, K^{-1} T^{*} K T u\right] \leq C_{\mathrm{I}}\left(\|u\|_{r, \Sigma}\|\partial(q T u)\|_{0, \Sigma}+\|u\|_{r, \Sigma}^{2}\right) .
$$

(II) Next

$$
\begin{aligned}
\operatorname{II}[T u, T u] & =(T \delta u, K T u)_{\tau}+([\delta, T] u, K T u)_{\tau} \\
& =\left(\delta u, K K^{-1} T^{*} K T u\right)_{\tau}+([\delta, T] u, K T u)_{\tau} .
\end{aligned}
$$


Therefore we have

$$
\mathrm{II}[T u, T u]-\mathrm{II}\left[u, K^{-1} T^{*} K T u\right]=([\delta, T] u, K T u)_{\tau} \leq C_{\mathrm{II}}\|u\|_{r, \tau}^{2}
$$

(III) By the same way as (II) we have

$$
\operatorname{III}[T u, T u]-\operatorname{III}\left[u, K^{-1} T^{*} K T u\right] \leq C_{\mathrm{III}}\|u\|_{r-1, \tau}\|T u\|_{0, \tau} .
$$

(IV) Finally

$$
\operatorname{IV}[T u, T u]-\operatorname{IV}\left[u, K^{-1} T^{*} K T u\right]=0 .
$$

Thus (I), (II), (III) and (IV) conclude the proposition.

Now, by using (8), we shall estimate the term $P_{t}^{s}\left[u, K^{-1} T^{*} K T u\right]$ with $u=u_{\varepsilon}$ which was introduced in Proposition 2. That is,

Proposition 4. We have, with a suitable constant $C>0$,

$$
\begin{aligned}
\left|P_{t}^{\varepsilon}\left[u_{\varepsilon}, K^{-1} T^{*} K T u_{\varepsilon}\right]\right| \leq & C\left(\left\|p_{\varepsilon} \partial^{r-1} f\right\|_{0, \Sigma}\left\|p_{\varepsilon} \partial\left(K T u_{\varepsilon}\right)\right\|_{0, \Sigma}\right. \\
& \left.+\|f\|_{r-2+1 / 2, \Sigma}\left\|T u_{\varepsilon}\right\|_{1 / 2, \Sigma}+\|\varphi\|_{r, \Sigma}\left\|T u_{\varepsilon}\right\|_{0, \tau}\right) .
\end{aligned}
$$

Proof. For the sake of simplicity, we write $u_{s}=u$. Then

$$
\begin{aligned}
P_{t}^{\epsilon}\left[u, K^{-1} T^{*} K T u\right]= & \left(J q_{\varepsilon} f, K^{-1} T^{*} K T u\right)_{\Sigma}+\left(\varphi, K K^{-1} T^{*} K T u\right)_{\tau} \\
= & \left(\zeta K^{-1} J q_{\varepsilon} f, \partial^{\rho} K T u\right)_{\Sigma}+\left(\varphi, T^{*} K T u\right)_{\tau} \\
= & \left(\zeta K^{-1} J q_{\varepsilon}(-\partial)^{\rho^{\prime}} f, \partial K T u\right)_{\Sigma}+\left(\left[(-\partial)^{\rho^{\prime}}, \zeta K^{-1} J q_{\varepsilon}\right] f, \partial(K T u)\right)_{\Sigma} \\
& +\left(\varphi, T^{*} K T u\right)_{\tau} \quad\left(\partial^{\rho}=\partial^{\rho^{\prime}} \partial\right) \\
= & \left(\zeta K^{-1} J p_{\varepsilon}(-\partial)^{\rho^{\prime}} f, p_{\varepsilon} \partial(K T u)\right)_{\Sigma} \\
& -\left(\partial\left[(-\partial)^{\rho^{\prime}}, \zeta K^{-1} J q_{\varepsilon}\right] f, K T u\right)_{\Sigma}+\left(\varphi, T^{*} K T u\right)_{\tau},
\end{aligned}
$$

from which we easily obtain the proposition.

Proposition 5. There exists a constant $C_{0}>0$ such that

$$
\begin{aligned}
\| p_{\varepsilon} \partial^{r+1} & u_{\varepsilon}\left\|_{0, \Omega}^{2}+(1+t)\right\| u_{\varepsilon} \|_{r, \Gamma}^{2} \\
\leq & C_{0}\left(\left\|u_{\varepsilon}\right\|_{r, \Omega}^{2}+\sum_{s=0}^{r-1}\left\|p_{\varepsilon} \partial^{s} f\right\|_{0, \Omega}^{2}+\|f\|_{r-2+1 / 2, \Omega}\left\|u_{\varepsilon}\right\|_{r+1 / 2, \Omega}+\|\varphi\|_{r, \Gamma}^{2}\right. \\
& \left.+C_{\mathrm{II}}\left\|u_{\varepsilon}\right\|_{r, \Gamma}^{2}\right) .
\end{aligned}
$$

Proof. Using (13) and Proposition 3 with $u=u_{\mathrm{s}}$, we can obtain, with the aid of Proposition 4.

$$
\begin{aligned}
& \left\|p_{\varepsilon} \partial T u_{\varepsilon}\right\|_{0, \Sigma}^{2}+\left\|p_{\varepsilon} T u_{\varepsilon}\right\|_{0, \Sigma}^{2}+(1+t)\left\|T u_{\varepsilon}\right\|_{0, \tau}^{2} \\
& \leq C_{1}\left(\left\|u_{\varepsilon}\right\|_{r, \Omega}^{2}+\sum_{s=0}^{r-1}\left\|p_{\varepsilon} \partial^{s} f\right\|_{0, \Omega}^{2}+\|f\|_{r-2+1 / 2, \Omega}\left\|u_{\varepsilon}\right\|_{r+1 / 2, \Omega}+\|\varphi\|_{r,, \Gamma}^{2}\right. \\
& \quad+C_{\mathrm{II}}\left(\left\|u_{\varepsilon}\right\|_{r, \Gamma}^{2}\right) \quad\left(=C_{1} F\right) .
\end{aligned}
$$


Noting that this remains valid for any $\rho=\left(\rho_{1}, \cdots, \rho_{l-1}\right)$ with $|\rho| \leq r$, we have, with a suitable constant $C_{2}$,

$$
\sum_{|\rho| \leq r}\left(\left\|p_{\varepsilon} \partial^{\rho} \partial\left(\zeta u_{\varepsilon}\right)\right\|_{0, \Sigma}^{2}+\left\|p_{\varepsilon} \zeta u_{\varepsilon}\right\|_{0, \Sigma}^{2}+(1+t)\left\|\partial^{\rho}\left(\zeta u_{\varepsilon}\right)\right\|_{0, \tau}^{2}\right) \leq C_{2} F
$$

With the aid of (11), we can assert that $\partial_{l}^{2}\left(\zeta u_{\varepsilon}\right)$ can be written by a linear conbination of $\partial_{j} \partial_{l}\left(\zeta u_{\varepsilon}\right), \partial_{j} \partial_{k}\left(\zeta u_{\varepsilon}\right)(j, k=1, \cdots, l-1), \partial_{j}\left(\zeta u_{s}\right)(j=1$, $\cdots, l), \zeta u_{\varepsilon}, \zeta f$ and $[A, \zeta] u_{\varepsilon}$. Hence we have

$$
\sum_{|\rho| \leq r-1}\left\|p_{\varepsilon} \partial^{\rho} \partial^{2}\left(\zeta u_{\varepsilon}\right)\right\|_{0, \Sigma}^{2}+(1+t) \sum_{|\rho| \leq r}\left\|\partial^{\rho}\left(\zeta u_{\varepsilon}\right)\right\|_{0, \tau}^{2} \leq C_{3} F .
$$

Repeating this process if $r>1$, we finally obtain

$$
\left\|p_{\varepsilon} \partial^{r+1}\left(\zeta u_{\varepsilon}\right)\right\|_{0, \Sigma}^{2}+(1+t) \sum_{|\rho| \leq r}\left\|\partial^{\rho}\left(\zeta u_{\varepsilon}\right)\right\|_{0, \tau}^{2} \leq C_{4} F .
$$

Clearly this remains also valid for $\zeta=\zeta_{0}$. Therefore applying this for $\zeta=\zeta_{j}(j=0, \cdots, N)$ and using $\sum_{j=0}^{N} \zeta_{j}=1$ on $\bar{\Omega}$, we obtain

$$
\left\|p_{\varepsilon} \partial^{r+1} u_{\varepsilon}\right\|_{0, \Omega}^{2}+(1+t)\left\|u_{\varepsilon}\right\|_{r, \Gamma}^{2} \leq C_{5} F \text {. }
$$

This completes the proof.

Proposition 6. For every integer $k \geq 2$, we can find two constant $C_{k}>0$ and $t_{k} \geq 0$ such that

$$
\left\|p_{\varepsilon} \partial^{k} u_{\varepsilon}\right\|_{0, \Omega}^{2}+\left\|u_{\varepsilon}\right\|_{k-1 / 2, \Omega}^{2} \leq C_{k}\left(\left\|p_{\varepsilon} \partial^{k-2} f\right\|_{0, \Omega}^{2}+\|f\|_{k-2-1 / 2, \Omega}^{2}+\|\varphi\|_{k-1, \Gamma}^{2}\right)
$$

is valid for all $\varepsilon$ and $t \geq t_{k}$.

Proof. Using the preceding proposition in the case $k=r+1$ and $t \geq C_{0} C_{\mathrm{II}}\left(=t_{k}\right)$, we have

$$
\begin{aligned}
& \left\|p_{\varepsilon} \partial^{k} u_{\varepsilon}\right\|_{0, \Omega}^{2}+\left\|u_{\varepsilon}\right\|_{k-1, \Gamma}^{2} \\
& \quad \leq C\left(\left\|u_{\varepsilon}\right\|_{k-1, \Omega}^{2}+\sum_{s=0}^{k-2}\left\|p_{\varepsilon} \partial^{s} f\right\|_{0, \Omega}^{2}+\|f\|_{k-2-1 / 2, \Omega}\left\|u_{\varepsilon}\right\|_{k-1 / 2, \Omega}+\|\varphi\|_{k-1 \Gamma}^{2}\right) .
\end{aligned}
$$

From (11) and the coercive inequality for Dirichlet problem it follows

$$
C^{\prime}\left\|u_{\varepsilon}\right\|_{k-1 / 2, \Omega}^{2}-\|f\|_{k-2-1 / 2, \Omega}^{2} \leq\left\|u_{\varepsilon}\right\|_{k-1, \Gamma}^{2} .
$$

The interpolation inequality says that for any $\delta>0$ there exists a constant $C_{\delta}>0$ such that

$$
\|u\|_{k-1, \Omega}^{2} \leq \delta\|u\|_{k-1 / 2, \Omega}^{2}+C_{\delta}\|u\|_{0, \Omega}^{2}, \quad u \in C^{\infty}(\bar{\Omega}) .
$$

Thus, the inequalities (16), (17) and (18) together with (9) immedi- 
ately imply the proposition.

In the below, Theorem 1 will be proved. We begin with the proof in case $f \in C^{\infty}(\bar{\Omega})$ and $\varphi \in C^{\infty}(\Gamma)$. So that we can use Propositions 1-6. Proposition 6 becomes, by using the notation (12),

$$
\left\|u_{\varepsilon} ; p\right\|_{k} \leq C_{k}\left(\left\|f ; p_{\epsilon}\right\|_{k-2}+\|\varphi\|_{k-1, \Gamma}\right) .
$$

The theorem of Banach-Sacks guarantees that there exists a sequence $\varepsilon_{1}>\varepsilon_{2}>\cdots$ converging to zero such that, as $n \rightarrow \infty$,

$$
v_{n}=\frac{u_{\varepsilon_{1}}+\cdots+u_{\varepsilon_{n}}}{n} \rightarrow u \quad \text { in } H^{k}(\Omega ; p) .
$$

From (8) we have, setting $B_{\lambda}[u, v]=B[u, v]+\lambda(u, v)$,

$$
\begin{aligned}
Q_{\lambda, t}\left[v_{n}, v\right]+B_{\lambda}\left[\frac{\varepsilon_{1} u_{\varepsilon_{1}}+\cdots+\varepsilon_{n} u_{\varepsilon_{n}}}{n}, v\right] \\
=(q f, v)_{\Omega}+(\varphi, v)_{\Gamma}+\frac{\varepsilon_{1}+\cdots+\varepsilon_{n}}{n}(f, v)_{\Omega} .
\end{aligned}
$$

Noting that $v_{n} \rightarrow u$ and $\varepsilon_{n} u_{\varepsilon_{n}} \rightarrow 0$ in $H^{k-\frac{1}{2}}(\Omega)$ as $n \rightarrow \infty$, we can derive

$$
Q_{\lambda, t}[u, v]=(q f, v)_{\Omega}+(\varphi, v)_{\Gamma}, \quad v \in C^{\infty}(\bar{\Omega}),
$$

and hence the $u$ satisfies (4). Moreover

$$
\begin{aligned}
\left\|v_{n} ; p\right\|_{k} & \leq \frac{1}{n}\left(\left\|u_{\varepsilon_{1}} ; p\right\|_{k}+\cdots+\left\|u_{\varepsilon_{n}} ; p\right\|_{k}\right) \\
& \leq C_{k}\left(\|f ; p\|_{k-2}+\|\varphi\|_{k-1, \Gamma}+\frac{\sqrt{\varepsilon_{1}}+\cdots+\sqrt{\varepsilon_{n}}}{n}\left\|\partial^{k-2} f\right\|_{0, \Omega}\right) .
\end{aligned}
$$

Accordingly, we obtain (5) as $n \rightarrow \infty$. It is easily seen that the uniqueness of solution of (4) follows from (19) and Proposition 1.

Suppose now that $f$ and $\varphi$ are in $H^{k-2}(\Omega ; p)$ and $H^{k-1}(\Gamma)$, respectively. Let $f_{j} \in C^{\infty}(\bar{\Omega})$ and $\varphi_{j} \in C^{\infty}(\Gamma)(j=1,2, \cdots)$ such that $f_{j} \rightarrow f$ in $H^{k-2}(\varphi ; p)$ and $\varphi_{j} \rightarrow \varphi$ in $H^{k-1}(\Gamma)$ as $j \rightarrow \infty$. For each $j$, we can find $u_{j} \in H^{k}(\Omega ; p)$ whose existence has just been proved, satisfying (4) and (5) with $f=f_{j}$ and $\varphi=\varphi_{j}$. We can immediately see that $u_{j}$ converges to $u$ in $H^{k}(\Omega ; p)$ an $j \rightarrow \infty$. Thus we finally obtain that $u$ is the unique solution of (4) and satisfies (5).

\section{§3. Proof of Corollary.}

Assume that there exists an open neighbourhood $U_{0}$ of $\Gamma_{0}$ in $\boldsymbol{R}^{l}$ such 
that $\gamma=0$ in $V_{0}=\Gamma \cap U_{0}$, and that $\left(\Gamma-V_{0}\right) \cap U_{j}$ is transformed by $\kappa_{j}$ to $\tau_{j}^{\prime} \subset \tau_{j}$. Then we have instead of (15)

$$
\left|([\delta, T] u, K T u)_{\tau}\right| \leq C_{\mathrm{II}}\|u\|_{r, \tau^{\prime}}^{2} .
$$

Hence we can change, in Proposition 5, the term $\left\|u_{\varepsilon}\right\|_{r, \Gamma}$ into $\left\|u_{\varepsilon}\right\|_{r, \Gamma-V_{0}}$. By the well known inequalities:

$$
\begin{aligned}
\|u\|_{r, \Gamma-V_{0}} & \leq \text { const. }\|u\|_{r+1 / 2, \Omega-U_{0}} \\
& \leq \delta\|u\|_{r+1, \Omega-U_{0}}+C_{\delta}\|u\|_{r, \Omega} \\
& \leq C\left(\delta\left\|p_{\delta} \partial^{r+1} u\right\|_{0, \Omega}+C_{\delta}\|u\|_{r, \Omega}\right),
\end{aligned}
$$

we obtain Proposition 5 with $C_{\mathrm{II}}=0$. In this case we have $t_{k}=0$ in Proposition 6. Thus we can assert Corollary.

\section{§4. Proof of Theorem 2.}

We assume that $\Gamma_{0}=\{x \in \Gamma ; \alpha(x)=0\}$ is a $C^{\infty}$ manifold of dimension $l-2$ and $\gamma$ is transversal to $\Gamma_{0}$. Let $U_{j}, \kappa_{j}, \Sigma_{j}, \tau_{j}, J_{j}, K_{j}$ and $\zeta_{j}$ be the same in $\S 1$. Here we further assume that for every $j$ such that $U_{j} \cap \Gamma_{0} \neq \emptyset$, the set $U_{j} \cap \Gamma_{0}$ is transformed onto an open portion $\tau_{j}^{0}$ of $y_{l}=0, y_{1}=0$ and $\gamma$ is altered to $\delta_{j}=\partial_{1}$ by $\kappa_{j}$, and $\gamma\left(\zeta_{j}(x)\right)=0$ in a neighbourhood $V_{0}$ of $\Gamma_{0}$.

LEMMA 4. There exists a positive $C^{\infty}$ function $h$ on $\Gamma$ such that

$$
\frac{1}{2} \gamma^{*}(h)+\beta h>0 \quad \text { on } \Gamma_{0} .
$$

Proof. By Lemma 1, we have only to find $h$ such that $-\gamma h+$ $(b+2 \beta) h>0$ on $\Gamma_{0}$. For every $j$ such that $U_{j} \cap \Gamma_{0} \neq \emptyset$, let $h_{j}$ be satisfying $-\partial_{1} h_{j}+(b+2 \beta) h_{j}=1$. Then $h=\Sigma \zeta_{j} h_{j}$ is a desired one, since $\gamma \zeta_{j}=0$ on $\Gamma_{0}$.

Using this lemma, we can easily prove

Lemma $2 '$. We can find a function $q(x) \in C^{\infty}(\bar{\Omega})$ satisfying

(i ) $q>0$ in $\Omega$ and $q=h \alpha$ on $\Gamma$.

(ii) (ii) of Lemma 2.

(iii) There exists a positive constant $c_{1}$ such that

$$
\frac{1}{2} \partial_{\nu} q+\frac{1}{2} \gamma^{*}(h)+\beta h \geq c_{1} \quad \text { on } \Gamma \text {. }
$$

If we define as 


$$
Q[u, v]=B[u, q v]+\int_{\Gamma}(h \gamma u+h \beta u) v d \sigma,
$$

then Propositions 1 and 2 with $t=0$ remain valid. We shall now show that Proposition 3 also holds if $P_{t}^{\epsilon}\left[u, K^{-1} T^{*} K T u\right]$ and $C_{\mathrm{II}}\|u\|_{r, \tau}^{2}$ are replaced with $P_{t}^{\epsilon}\left[u,(h K)^{-1} T^{*} h K T u\right]$ and $C_{\text {II }}\|u\|_{r, \tau^{\prime}}^{2}$, where $\tau^{\prime}$ denotes the same notation as in $\S 3$. In (I) of the proof of Proposition 3 we have only to replace $K$ with $h K$. In this case, the forms II and III become

$$
\mathrm{II}[u, v]=\int_{\tau} \partial_{1} u \cdot h K v d \sigma
$$

and

$$
\mathrm{III}[u, v]=\int_{\tau} \beta u \cdot h K v d \sigma
$$

Therefore we have

$$
\begin{aligned}
\mathrm{II}[T u, T u] & =\left(\partial_{1} T u, h K T u\right)_{\tau} \\
& =\left(T \partial_{1} u, h K T u\right)_{\tau}+\left(\left[\partial_{1}, T\right] u, h K T u\right)_{\tau} \\
& =\left(\partial_{1} u, h K(h K)^{-1} T^{*} h K T u\right)_{\tau}+\left(\left[\partial_{1}, T\right] u, h K T u\right)_{\tau} \\
& =\mathrm{II}\left[u,(h K)^{-1} T^{*} h K T u\right]+\left(\left[\partial_{1}, T\right] u, h K T u\right)_{\tau} .
\end{aligned}
$$

Hence

$$
\mathrm{II}[T u, T u]-\mathrm{II}\left[u,(h K)^{-1} T^{*} h K T u\right] \leq C_{\mathrm{II}}\|u\|_{r, \tau^{\prime}}^{2},
$$

since $\partial_{1} \zeta=0$ in $V_{0}$. It is obvious that

$$
\operatorname{III}[T u, T u]-\operatorname{III}\left[u,(h K)^{-1} T^{*} h K T u\right] \leq C_{\mathrm{III}}\|u\|_{r-1, \tau}\|T u\|_{0, \tau} .
$$

Thus, Proposition 3 can be concluded in our case.

By the same argument as in $\S 3$, we obtain Proposition 5 with $C_{\text {II }}=0$. Finally we can complete the proof of Theorem 2 by the same argument as in the proof of Theorem 1.

\section{REFERENCES}

[ 1 ] Ju. V. Egorov, V. A. Kondrat'ev, The oblique derivative problem, Mat. Sbornik 78 (1969), 148-176.= Math. USSR Sbornik 7 (1969), 139-169.

[2] K. Hayashida, on the singular boundary value problem for elliptic equations, Trans. Amer. Math. Soc., 184 (1973), 205-221.

[ 3 ] Y. Kato, Mixed-type boundary conditions for second order elliptic differential equations, to appear in J. Math. Soc. Japan 26 (1974). 
[4] V. G. Mazja, The degenerate problem with oblique derivative, Uspehi Mat. Nauk $25(1970), 275-276$.

Mathematical Institute

Nagoya University 